\title{
Optimizing the management of aneurysmal subarachnoid hemorrhage: Lessons learned and future directions
}

Sodhi and colleagues present the outcomes of patients presenting with aneurysmal subarachnoid hemorrhage (aSAH) at a high-volume, tertiary center in north India. ${ }^{[1]}$ This prospective cohort study with 482 patients was conducted over a period of 10 months. A total of 330 patients underwent treatment with neurosurgical clipping $(N=307)$ or endovascular coil embolization $(N=23)$. In the subgroup, which underwent received neurosurgical clipping, the 3-month mortality was $27.7 \%$ and $50.2 \%$ had good outcome, defined as a Glasgow Outcome Score (GOS) of 4 or 5. In the coiling subgroup, the 3 -month mortality was $17.3 \%$ and $73.9 \%$ had good outcome (GOS 4 or 5). The nonintervention cohort comprised 152 patients who did undergo aneurysm occlusion and only received supportive care. In this nonintervention cohort, the mortality rate was $85.5 \%$ at 3 months.

Multinomial regression analysis identified World Federation of Neurosurgical Societies (WFNS) grade and a history of hypertension to be independent predictors of poor outcome. The authors suggest that screening programs should be implemented in developing countries, such as India, to help detect and treat intracranial aneurysms before rupture. It would be interesting to know the morbidity and mortality rate of elective aneurysm treatment at the authors' institution. With this information, one might extrapolate the potential benefit of screening programs for detecting unruptured aneurysms. Of note, only $7 \%$ of patients in this study's intervention group underwent endovascular coiling, which is presumably due the limited availability of endovascular technology as well as the limited financial resources of a large hospital in

\begin{tabular}{|l|l|}
\hline \multicolumn{2}{|c|}{ Access this article online } \\
\hline Quick Response Code: & Website: \\
\hline & www.ruralneuropractice.com \\
\cline { 2 - 2 } & \\
\hline
\end{tabular}

a developing nation. Overall, this study emphasizes the importance of two longstanding principles of managing aSAH, which are largely taken for granted in Western societies: (1) ruptured aneurysms must be secured to prevent the catastrophic consequences associated with rebleeding and (2) aggressive critical care medicine must be performed in the 10-14 days following the ictus of aSAH in order to minimize the risk of delayed neurological and medical morbidity, which accompanies aneurysm rupture.

\section{Lessons Learned: Clipping Versus Coiling of Ruptured Aneurysms}

The International Subarachnoid Aneurysm Trial (ISAT) was a multicenter randomized controlled trial (RCT) comprised of 2143 patients, of which 1070 were assigned to clipping and 1073 were assigned to coiling. ${ }^{[2]}$ The primary outcome (functional dependency or death as assessed by modified Rankin Scale score of 3-6) was observed in $24 \%$ of patients in the coiling cohort compared with $31 \%$ of the patients in the clipping cohort $(P=0.002)$. A decade later, the barrow ruptured aneurysm trial (BRAT), a single institution RCT of 349 patients, of which 179 underwent clipping and 170 underwent coiling, reported superior outcomes with coiling at 1 year follow-up but statistically similar between the two therapeutic approaches at 3 years follow-up. ${ }^{[3,4]}$ At 3 years follow-up, the rates of poor outcome, defined as a modified Rankin Scale score greater than 2, in patients who underwent clipping and coiling were $35.8 \%$ and $30 \%$, respectively $(P=0.25) \cdot{ }^{[4]} \mathrm{Li}$ et al. performed a meta-analysis of 4 RCTs and 23 observational studies in order to compare clipping to coiling outcomes for ruptured aneurysms. ${ }^{[5]}$ The results showed superior clinical outcomes with coiling with a greater benefit for patients with better preoperative neurological grade but a lower risk of rebleeding with clipping. While coiling is the preferred approach for the majority of ruptured aneurysms, clipping remains an important treatment 
for aneurysms in which the risk of long-term recurrence following endovascular treatment is high.

\section{Lessons Learned: Management of Patients with Aneurysmal Subarachnoid Hemorrhage}

Traditionally, the care of aSAH patients after the initial phase of aneurysm treatment was focused on the prevention of cerebral vasospasm $(\mathrm{CV})$, which is detected radiographically and clinically in up to $70 \%$ and $30 \%$ of patients, respectively. However, emerging evidence, primarily from the repeated failures of various therapies aimed at abrogating aSAH-induced CV, has suggested causes of delayed cerebral ischemia (DCI) following aSAH, which are independent of vasospasm. ${ }^{[6]}$ Oral nimodipine is the pharmacologic agent that has been definitively proven to reduce the incidence of DCI following aSAH. ${ }^{[7]}$ Induced hypertension, hypervolemia, and hemodilution,

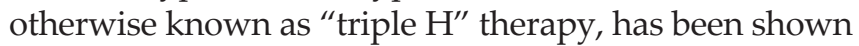
to have the ability to reverse the neurological deficits associated with clinically evident CV. ${ }^{[8]}$ Therefore the routine administration of nimodipine and the use of

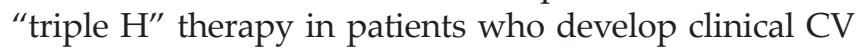
comprise the backbone of the critical care approach to any aSAH patient.

\section{Future Directions}

While endovascular technology continues to advance at a breakneck pace, it is unknown if any of these advances will improve outcomes in patients with ruptured aneurysms. Furthermore, it is unknown if new endovascular technologies will be practical for institutions in developing countries to acquire given the differences in the healthcare, financial, and government systems of these nations compared with Western countries. Nevertheless, it is clear that efforts are needed at a global level to diminish the persistently high rates of morbidity and mortality associated with aSAH.

\author{
Thomas J. Buell, Dale Ding \\ Department of Neurological Surgery, University of Virginia, \\ Charlottesville, Virginia, United States \\ Address for correspondence: \\ Dr. Dale Ding, \\ Department of Neurological Surgery, University of Virginia, \\ PO Box - 800212, Charlottesville, Virginia - 22908, United States. \\ E-mail: dmd7q@hscmail.mcc.virginia.edu
}

\section{References}

1. Sodhi HB, Savardekar A, Mohindra S, Chhabra R, Gupta V, Gupta SK. The clinical profile, management, and overall outcome of aneurysmal subarachnoid hemorrhage at the neurosurgical unit of a tertiary care center in India. J Neurosci Rural Pract 2014;5:118-26.

2. Molyneux A, Kerr R, Stratton I, Sandercock P, Clarke M, Shrimpton J, et al. International Subarachnoid Aneurysm Trial (ISAT) Collaborative Group. International Subarachnoid Aneurysm Trial (ISAT) of neurosurgical clipping versus endovascular coiling in 2143 patients with ruptured intracranial aneurysms: A randomised trial. Lancet 2002;360:1267-74.

3. McDougall CG, Spetzler RF, Zabramski JM, Partovi S, Hills NK, Nakaji P, et al. The barrow ruptured aneurysm trial. J Neurosurg 2012;116:135-44.

4. Spetzler RF, McDougall CG, Albuquerque FC, Zabramski JM, Hills NK, Partovi S, et al. The barrow ruptured aneurysm trial: 3-year results. J Neurosurg 2013;119:146-57.

5. Li H, Pan R, Wang H, Rong X, Yin Z, Milgrom DP, et al. Clipping versus coiling for ruptured intracranial aneurysms: A systematic review and meta-analysis. Stroke 2013;44:29-37.

6. Macdonald RL. Delayed neurological deterioration after subarachnoid haemorrhage. Nat Rev Neurol 2014;10:44-58.

7. Allen GS, Ahn HS, Preziosi TJ, Battye R, Boone SC, Boone SC, et al. Cerebral arterial spasm-a controlled trial of nimodipine in patients with subarachnoid hemorrhage. N Engl J Med 1983;308:619-24.

8. Kassell NF, Peerless SJ, Durward QJ, Beck DW, Drake CG, Adams HP. Treatment of ischemic deficits from vasospasm with intravascular volume expansion and induced arterial hypertension. Neurosurgery 1982;11:337-43.

How to cite this article: Buell TJ, Ding D. Optimizing the management of aneurysmal subarachnoid hemorrhage: Lessons learned and future directions. J Neurosci Rural Pract 2014;5:109-10.

Source of Support: Nil. Conflict of Interest: None declared. 